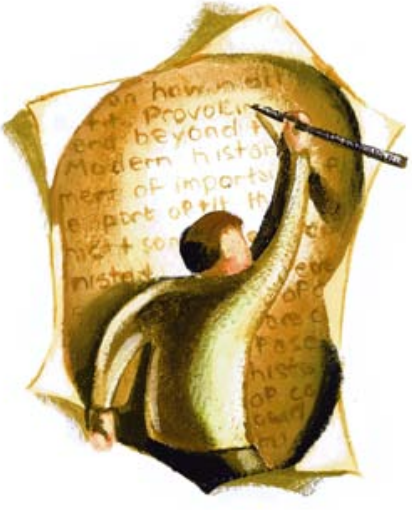

\section{Not-so-surprising findings}

Investigators who publish their research in medical journals usually report effect measures in the form of ratios, such as relative risk or rate ratio. It is well known that the magnitude of ratio measures depends on the underlying risk for the study outcome: ${ }^{1}$ the higher the baseline risk in a population, the lower the risk ratio for a constant excess risk (Fig. I). Randomized controlled trials (RCTs) often include only low-risk patients, selected through stringent exclusion criteria, wheras epidemiologic studies usually include typical patients with multiple comorbidities. These differences in baseline risk lead to higher ratio effect measures in RCTs than in nonrandomized studies, which would explain some of the observations reported by Panagiotis Papanikolaou and associates. ${ }^{2}$

Of relevance to this point is their scenario 15 on the risk of myocardial infarction in the context of therapy with rofecoxib or naproxen. ${ }^{2}$ Table I shows the difference in risk structure and baseline event rates between the VIGOR trial ${ }^{3}$ (which focused on gastrointestinal effects and excluded patients with major cardiovascular conditions) and the nonrandomized study by Ray and colleagues. ${ }^{4}$ These differences resulted in numerically higher rate ratio measures in the RCT (because of the lower baseline event rate), despite smaller rate differences.

In meta-analytic comparisons between RCTs and nonrandomized studies that fail to adjust for the re-

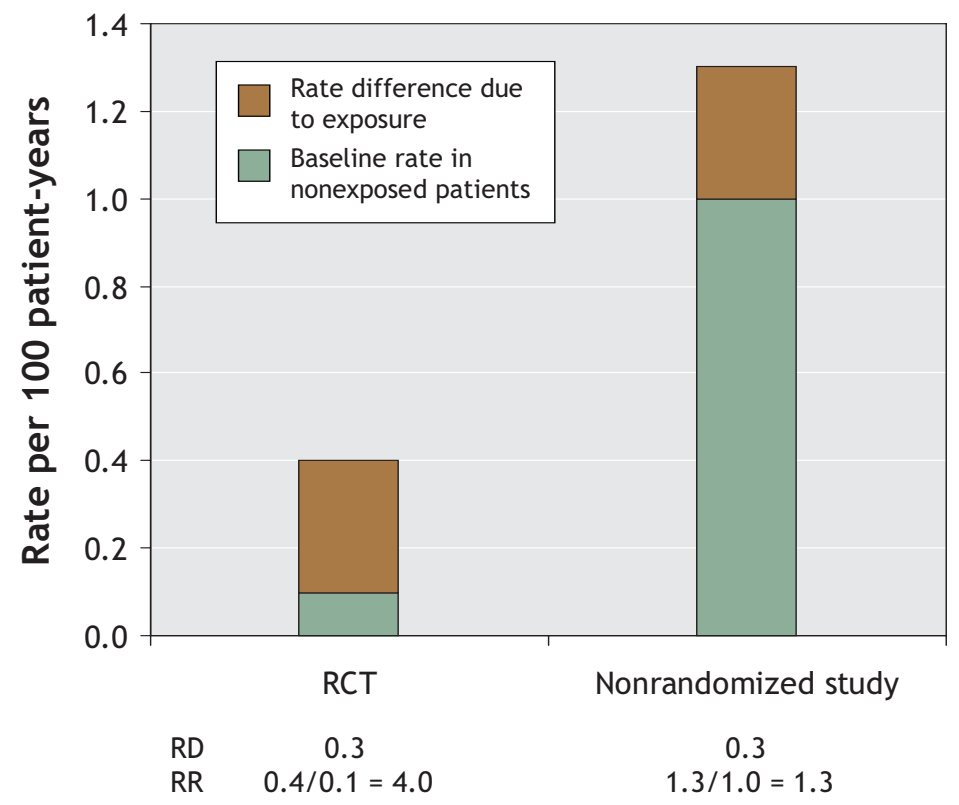

Fig. 1: Illustration of the decrease in rate ratio (RR) with an increasing baseline outcome rate when the rate difference (RD) is constant (at 0.3 per 100 patient-years) but the baseline rate in unexposed patients differs (0.1 or 1 per 100 patient-years). The bar at left represents data from the VIGOR trial. $^{3}$

Table 1: Comparison of data from a randomized trial and epidemiologic study of acute myocardial infarction among users of naproxen or rofecoxib

\begin{tabular}{|c|c|c|}
\hline Characteristic & VIGOR $^{3}$ & Ray et al. ${ }^{4}$ \\
\hline Study type & RCT & Cohort study \\
\hline Age of patients (yr) & $\geq 40$ & $\geq 50$ \\
\hline Population & Volunteers & $\begin{array}{c}\text { Medicaid } \\
\text { (very low income) }\end{array}$ \\
\hline $\begin{array}{l}\% \text { of patients in naproxen group } \\
\text { with major cardiovascular disease } \\
\text { (IHD, acute MI, stroke, CHF) }\end{array}$ & Not reported* & $38 \%$ \\
\hline \multicolumn{3}{|l|}{ Rate of acute MI } \\
\hline $\begin{array}{l}\text { Among naproxen users } \\
\text { (per } 100 \text { patient-years) }\end{array}$ & 0.1 & 1.11 \\
\hline $\begin{array}{l}\text { Mmong rofecoxib users } \\
\text { (per } 100 \text { patient-years) }\end{array}$ & 0.4 & 2.40 \\
\hline Unadjusted rate difference & 0.3 & 1.29 \\
\hline Unadjusted rate ratio & 4.0 & 2.16 \\
\hline Multivariate adjusted rate ratio & NA & $2.10 \dagger$ \\
\hline
\end{tabular}

$\mathrm{RCT}=$ randomized controlled trial, IHD = ischemic heart disease, $\mathrm{MI}=$ myocardial infarction, $\mathrm{CHF}=$ congestive heart failure.

*The VIGOR trial ${ }^{3}$ excluded patients with a history of cerebrovascular events in the 2 years before the study and those with acute MI or coronary artery bypass grafting in the year before the study. Numbers of these baseline risk factors were not presented but can be presumed to be substantially lower than those in the study by Ray and associates. ${ }^{4}$

†Not reported but approximated as 1.93 (adjusted rofecoxib effect in $>25 \mathrm{mg}$ group) divided by 0.92 (adjusted naproxen effect). 
spective baseline risks, the interpretation of ratio effect measures may be misleading. It has previously been demonstrated that careful epidemiologic studies that mimic the exclusion criteria of RCTs are likely to result in the same effect sizes as the RCTs. ${ }^{5}$ The strength of many nonrandomized studies is their assessment of harms of medical interventions in populations that are usually excluded from RCTs.

\section{Sebastian Schneeweiss \\ Daniel H. Solomon}

Division of Pharmacoepidemiology

and Pharmacoeconomics

Department of Medicine

Brigham and Women's Hospital

Harvard Medical School

Boston, Mass.

\section{REFERENCES}

I. Barratt A, Wyer PC, Hatala R, et al; Evidence-Based Medicine Teaching Tips Working Group. Tips for learners of evidence-based medicine: I. Relative risk reduction, absolute risk reduction and number needed to treat. CMAJ 2004;I7I(4):353-8.

2. Papanikolaou PN, Christidi GD, Ioannidis JPA. Comparison of evidence on harms of medical interventions in randomized and nonrandomized studies. CMAJ 2006;174(5):635-4I.

3. Bombardier C, Laine L, Reicin A, et al; VIGOR Study Group. Comparison of upper gastrointestinal toxicity of rofecoxib and naproxen in patients with rheumatoid arthritis. $N$ Engl $\mathrm{J} \mathrm{Med}$ 2000;343:I520-8.

4. Ray WA, Stein CM, Daugherty JR, et al. COX-2 selective non-steroidal anti-inflammatory drugs and risk of serious coronary heart disease. Lancet 2002;360:107I-3.

5. Britton A, McKee M, Black N, et al. Choosing between randomised and non-randomised studies: a systematic review. Health Technol Assess I998;2(I3);i-iv, I-I24.

DOI:Io.1503/cmaj.1060ogo

\section{[Two of the authors respond:]}

We thank Schneeweiss and Solomon for their interesting comment. We fully agree that nonrandomized studies often include high-risk populations that are excluded from randomized trials. However, this is not an absolute rule. While some of the discrepancies between absolute and relative effect metrics may be explained by differences in baseline risk, this has to be checked on a case-by-case basis. It is also very difficult to reach consensus whether harmful effects are described more appropriately in the absolute or multiplicative scale, so we opted to show both in our evaluation. ${ }^{1}$ Besides genuine differences in absolute risk, measurement problems and bias should also be considered. Absolute event rates may vary considerably across studies, regardless of design, because of many reasons. These include differences in the definition of the adverse event; the captured range of severity; the threshold of patients and physicians to report (often a reflection to the extent to which they are sensitized); the mode of data collection (in particular, active versus passive surveillance for harms); and whether any efforts at attribution have been made. ${ }^{2,3}$ In the absence of standardization of collection and reporting of information, ${ }^{4}$ comparisons of absolute event rates may sometimes remain tenuous. Therefore, while absolute event rates are clinically most meaningful and can be readily translated to numbers needed to harm, relative risks may be somehow more robust.

\section{John P.A. Ioannidis}

Panagiotis Papanikolaou

Department of Hygiene

and Epidemiology

University of Ioannina School

of Medicine

Ioannina, Greece

\section{REFERENCES}

I. Papanikolaou PN, Christidi GD, Ioannidis JPA. Comparison of evidence on harms of medical interventions in randomized and nonrandomized studies. CMAJ 2006;174(5):635-4I.

2. Bent S, Padula A, Avins AL. Better ways to question patients about adverse medical events: a randomized, controlled trial. Ann Intern Med 2006; I44:257-6I.

3. Ioannidis JP, Mulrow CD, Goodman SN. Adverse events: the more you search, the more you find. Ann Intern Med 2006;144:298-300.

4. Ioannidis JP, Evans SJ, Gotzsche PC, et al, CONSORT Group. Better reporting of harms in randomized trials: an extension of the CONSORT statement. Ann Intern Med 2004;14I:78I-8.

DOI:I0.I503/cmaj.Io6oII3

\section{Virtual links to the}

\section{emergency department}

Eddy Lang and colleagues were quite optimistic in their expectations of the power of communication between the emergency department (ED) and family physicians. ${ }^{1}$ We all want to reduce du- plication and unnecessary admissions to hospital. These benefits of an electronic communications system, however, would not attract me as a practising family doctor. Instead, the benefits I find useful are the time saved in not having to hound hospitals for information and the increased comfort I would feel in knowing what had actually happened to my patient in the ED. Family physicians are leaving their practice in droves and having timely information to make clinical decisions is one factor that may make family practice more palatable.

\section{Laura K. Muldoon \\ Family Physician \\ Ottawa, Ont.}

\section{REFERENCE}

I. Lang $\mathrm{E}$, Afilalo $\mathrm{M}$, Vandal AC, et al. Impact of an electronic link between the emergency department and family physicians: a randomized controlled trial. $C M A J$ 2006;174(3):313-8.

Conflict of Interest: None declared.

DOI:I0.1503/cmaj.I060055

We wish to congratulate the authors for this well done study on an important research question: they found that an electronic link between an ED and family physicians produced no effect. ${ }^{1}$

In eHealth, failure to use technology is frequently observed, and is an important outcome. ${ }^{2}$ The authors of this study should report access and usage by the family physicians; if the communication software was infrequently used, it would not have changed outcomes.

Our second area of concern is the choice of family physicians eligible to participate. The authors chose physicians with the highest number of patient visits to emergency; the 43 eligible physicians likely represent about Io\% of all family physicians at their institution. Comparing their characteristics with those of their peers may be worthwhile. The average practice size for physicians in the study is $4 \mathrm{I} 84$ patients. ${ }^{1}$ In Ontario, the Family Health Network contract limits groups to an average practice size of 2400 patients for full payment. Partic- 